\section{Violência no trabalho em saúde: a experiência de servidores estaduais da saúde no Estado da Bahia, Brasil}

\author{
Workplace violence in the healthcare sector: \\ the experience of State health employees in \\ Bahia State, Brazil
}

\author{
Violencia en el trabajo en el ámbito de salud: \\ la experiencia de los empleados de salud del \\ estado en Bahía, Brasil
}

Iracema Viterbo Silva 1,2

Estela M. L. Aquino 1

Isabela Cardoso de Matos Pinto ${ }^{1}$

\author{
${ }^{1}$ Instituto de Saúde Coletiva, \\ Universidade Federal da \\ Bahia, Salvador, Brasil. \\ 2 Superintendência de \\ Recursos Humanos, \\ Secretaria da Saúde do \\ Estado da Bahia, Salvador, \\ Brasil. \\ Correspondência \\ I. V. Silva \\ Instituto de Saúde Coletiva, \\ Universidade Federal da \\ Bahia. \\ Rua Basílio da Gama s/n, \\ Campus Universitário \\ Canela, Salvador, BA \\ 40110-040, Brasil. \\ iviterbos@hotmail.com
}

\begin{abstract}
This study aimed to estimate the prevalence of self-reported violence suffered by healthcare workers, using a cross-sectional design with a sample of 679 State health employees through face-to-face interviews and a questionnaire. Of the respondents, $25.9 \%$ (95\%CI: 22.6\%-29.2\%) reported having suffered at least one form of violence, with verbal aggression as the most frequent (19.4\%). Women aged 25-39 suffered $80 \%$ more violence than older women $(\mathrm{OR}=$ 1.8; 95\%CI: 1.1-3.0). Female physicians were the most frequently affected group $(O R=2.5$; 95\%CI: 1.2-12.5). Among men, a nearly fourfold increase in healthcare workplace violence was associated with age 25 to 39 years (OR = 3.9; 95\% CI: 1.916.4) and nurse assistants or nurse technicians (PR = 3.9; 95\%CI: 1.1-13.2). This study can help raise awareness concerning healthcare workplace violence and support policies for health workers' care, with repercussions on quality of care for health services users.
\end{abstract}

Workplace Violence; Health Personnel; Gender Identity

\section{Resumo}

Este estudo teve por objetivo estimar a prevalência de violência autorreferida no trabalho em saúde. Estudo transversal realizado com uma amostra de 679 servidores estaduais (Bahia, Brasil), por meio de entrevistas face a face e uso de questionário. Dos entrevistados, 25,9\% (IC95\%: 22,6\%-29,2\%) referiram pelo menos uma das modalidades de violência investigadas, sendo a agressão verbal $(19,4 \%)$ a mais frequente. As mulheres na faixa etária de 25 a 39 anos apresentaram um acréscimo de $80 \%$ na ocorrência de violência em relação às mais velhas $(\mathrm{OR}=1,8$; IC95\%: 1,1-3,0), enquanto que as médicas também foram as mais atingidas $(O R=2,5$; IC95\%: 1,2-12,5). Entre os homens, ter de 25 a 39 anos $(O R=3,9 ;$ IC95\%: 1,9-16,4) e trabalhar como auxiliar ou técnico em enfermagem ( $R P=3,9$; IC95\%: 1,1-13,2) aumentou quase quatro vezes a ocorrência de violência no trabalho em saúde. Este estudo pode trazer contribuições importantes para a visibilidade da violência no setor saúde e fornecer subsídios para a formulação de políticas de atenção aos trabalhadores com repercussão na qualidade do atendimento prestado à população.

Violência no Trabalho; Pessoal de Saúde; Identidade de Gênero 


\section{Introdução}

A violência no local de trabalho tem sido apresentada como um novo risco ocupacional, estando os trabalhadores da saúde particularmente expostos a este tipo de violência 1. É definida pela Organização Mundial da Saúde (OMS) como "o uso intencional da força, real ou em forma de ameaça, contra outra pessoa ou contra um grupo, em circunstâncias relacionadas com o trabalho que resulte ou possa resultar em lesão, morte, dano psicológico, mau desenvolvimento ou privação" 2 (p. 5).

Não obstante a abrangência dessa definição, inúmeros são os significados que podem ser encontrados na literatura acerca do fenômeno. Alguns consideram apenas os casos de ameaça ou agressão física, e outros, qualquer tipo de comportamento que venha a prejudicar os trabalhadores ou suas organizações 3,4 . As várias definições existentes acabam dificultando a estimativa da incidência do problema no setor, sendo importante a realização de estudos que deem visibilidade ao problema.

Ainda que presente em todos os setores ocupacionais, a violência no trabalho é considerada mais frequente nos espaços onde há predominância de mulheres, caso do setor de saúde e de serviços sociais 5 . Fato que sugere a influência das relações de gênero nessa determinação. De modo que, embora se atribua a ocorrência de violência entre as profissões consideradas mais perigosas, como a de vigilantes e policiais, os estudos têm chamado a atenção para a elevada prevalência do problema nas profissões tipicamente femininas, em particular as exercidas por mulheres mais jovens e com menor nível de escolaridade 6,7 .

No que tange à violência às mulheres, já existe vasta literatura que associa a vitimização às diferenças de gênero 8,9,10. Entretanto, apesar dos vários estudos sobre o assunto no espaço doméstico, presume-se que a violência contra as mulheres no local de trabalho, especialmente no que se refere ao assédio sexual, ainda permanece na invisibilidade 11 .

Em relação à violência física, o trabalho de Franz et al. 12, realizado na Alemanha, encontrou uma prevalência de $7,7 \%$, tendo os autores observado que profissionais com idade igual ou menor que 44 anos estavam mais sujeitos a sofrer esse tipo de agravo. A prevalência também foi maior para funcionários de hospital, tendo médicos, enfermeiros e trabalhadores de ambulância como as categorias que mais referiram essa modalidade. No mesmo estudo, a violência psicológica, expressa principalmente por meio de agressão verbal, apresentou uma prevalência de
$38,6 \%$, atingindo os vários grupos etários, porém um pouco maior entre as pessoas mais jovens.

Em relação aos agressores, na totalidade dos estudos, os pacientes aparecem como os principais perpetradores seguidos de seus familiares, mas observa-se que frequentemente a violência no espaço da saúde também ocorre por parte de membros da própria equipe 13 . Um trabalho realizado na Jamaica com profissionais de saúde, mostra que quase todos os atos de violência física (97\%) e psicológica (94\%) ocorreram dentro da instituição de saúde, sendo poucos os incidentes ocorridos no percurso do trabalho ou fora da unidade 14 .

Ainda que a violência seja um fenômeno que afeta praticamente todos os setores e categorias de trabalhadores, o setor saúde apresenta algumas singularidades. Muitos trabalhadores da saúde exercem suas atividades à noite, sozinhos ou de forma isolada, estando sujeitos a um risco maior de violência 15 . Por outro lado, é uma atividade na qual o trabalhador mantém uma relação direta com o seu objeto de trabalho que são as necessidades de saúde do indivíduo doente ou exposto a risco de adoecer, e sendo este indivíduo um ser humano permeado de subjetividade poderá interferir no trabalho que é realizado 16,17.

No Brasil, observa-se certa naturalização do fenômeno, na medida em que os profissionais mostram aceitar tais fatos como parte do cotidiano do trabalho, demonstrando certo desinteresse na tomada de providências. De acordo com os autores consultados, a não ser que ocorra um episódio considerado grave ou surjam ameaças contínuas, procedimentos formais de notificação são pouco conhecidos e utilizados pelos profissionais 18 .

A falta de notificação camufla o problema e prejudica os trabalhadores atingidos, a quem, segundo Lancman et al. ${ }^{19}$, não são oferecidos programas de prevenção e apoio devido à forma banal como a situação é encarada, o que acaba indicando certa omissão em relação ao problema. Acredita-se que uma política de não valorização do profissional da saúde aliada a uma sociedade em que não há punição para atos de violência acabe por desmotivar a denúncia e a busca por providências.

Essa naturalização do fenômeno também é observada nos estudos realizados em outros países. Na Jamaica, onde as equipes de saúde têm experimentado frequentemente algumas formas de violência física ou psicológica, o fenômeno é considerado pelos profissionais como "uma parte inevitável do trabalho", sendo encarado como situação normal 14 .

Apesar dessa naturalização, a violência no trabalho em saúde, como todo comportamento 
abusivo, é considerada pelos autores como um fator que leva ao adoecimento, medo, insatisfação e outros sentimentos de insegurança e desmotivação que trazem repercussões para a saúde do trabalhador 18. Muitos são os sintomas de ordem física, mental e comportamental decorrentes da situação de violência vivida no trabalho, podendo os trabalhadores experimentar medo, diminuição da autoconfiança, distúrbios do sono e irritabilidade, além de depressão e síndrome de estresse pós-traumático, em muito maior proporção para a violência não física 13 .

Considerando que ainda são poucas as pesquisas e insuficientes as informações sobre o assunto, além dos efeitos negativos que o problema traz para o trabalhador do segmento, foi realizado este estudo com o objetivo de estimar a prevalência de violência autorreferida no trabalho em saúde, identificando as modalidades mais comuns e os perfis sociodemográfico e ocupacional dos profissionais atingidos.

\section{Metodologia}

\section{Desenho de estudo}

Trata-se de um estudo de corte transversal que incluiu servidores estatutários lotados no nível central e nas unidades de saúde sob gestão direta da Secretaria da Saúde do Estado da Bahia (SESAB), localizados no Município de Salvador, Brasil. Foram incluídos os trabalhadores em atividade ou afastados temporariamente por problemas de saúde, licença prêmio ou férias. Foram inelegíveis os servidores cedidos a outros órgãos e secretarias estaduais, servidores que trabalham em unidades municipalizadas, federalizadas, fundações ou sob gestão terceirizada e aqueles lotados em unidades do interior do estado, bem como os aposentados e licenciados por interesse particular (licença sem vencimentos).

\section{Plano amostral}

Para a definição do tamanho amostral foi calculada uma amostra probabilística do tipo aleatória simples. Assumiu-se uma prevalência de $33 \%$, um erro aceitável de $5 \%$ e um poder de $80 \%$, ficando a amostra definida em 676 servidores. Considerando possíveis perdas ou recusas, esse número foi ampliado para 800 pessoas. $\mathrm{Na}$ seleção dos sujeitos realizada eletronicamente por meio do SPSS (SPSS Inc., Chicago, Estados Unidos) recorreu-se à lista de servidores lotados em unidades localizadas no Município de Salvador, fornecida pela SESAB, com um total de 13.390, utilizando como identificador o número da matrícula no órgão. Os casos inelegíveis encontrados durante a produção de dados foram substituídos por sujeitos com características similares que atenderam aos critérios de inclusão, enquanto que as recusas, óbitos e condições de saúde que impediram a realização da entrevista foram considerados como perdas.

\section{Produção de dados}

Os dados foram produzidos por meio de entrevista face a face, sendo utilizado um questionário composto de 195 questões, distribuídas em oito blocos. Além das características sociodemográficas e ocupacionais, o instrumento incluía um bloco sobre violência no local de trabalho, com episódios ocorridos nos últimos 12 meses.

Para a investigação da violência autorreferida no local de trabalho foram selecionadas as 19 questões do instrumento utilizado no estudo Violência no Local de Trabalho no Setor Saúde, versão em português e que abordavam cinco modalidades de violência, sua frequência e tipo de agressor. Essa pesquisa foi realizada em oito países, incluindo o Brasil, sendo uma iniciativa do International Labour Office (ILO), International Council of Nurses (ICN), OMS e Public Services International (PSI) ${ }^{15}$. O instrumento investigava o grau de preocupação do entrevistado com a violência no seu local de trabalho, de acordo com cinco níveis de respostas: bastante preocupado; muito preocupado; preocupado; pouco preocupado; e nada preocupado. Também buscava informações sobre os tipos de violência sofrida física, sexual e/ou psicológica, conforme definição utilizada: violência física entendida como o uso da força física contra outra pessoa ou grupo, que resulta em dano físico, sexual ou psicológico. Inclui bater, dar pontapé, esbofetear, alvejar, empurrar, morder, beliscar, contaminar com produtos orgânicos - saliva, sangue, entre outros.

A violência psicológica foi investigada de acordo com três modalidades: ameaça ou agressão verbal, discriminação e danos contra propriedade pessoal. Foi considerada ameaça ou agressão verbal "um comportamento que humilha, degrada ou indica uma falta de respeito pela dignidade e valor de uma pessoa. É a comunicação por palavras, tom ou postura que rebaixa, ameaça, acusa ou desrespeita o outro" 15 (p. 6). Como discriminação considerou-se "qualquer conduta ameaçadora baseada na raça, cor, linguagem, nacionalidade, religião, associação com uma minoria, nascimento ou outra característica que não é recíproca nem desejada e que afeta a dignidade das mulheres ou dos homens no trabalho" 15 (p. 6). No que se refere à violência sexual, investigou-se a ocorrência de assédio, en- 
tendendo-se como "qualquer comportamento de natureza sexual indesejado, não recíproco e mal recebido que é ofensivo para a pessoa envolvida que faz com que se sinta ameaçada, humilhada ou embaraçada" 15 (p. 6). Investigou-se, ainda, a frequência e a autoria da violência e se já havia testemunhado algum desses tipos de violência sendo praticado contra outra pessoa no seu local de trabalho.

O estudo foi realizado no período de dezembro de 2011 a julho de 2012, e à proporção que as entrevistas eram realizadas, os dados foram digitados utilizando-se o aplicativo Epi Data, versão 3.0 (Epi Data Assoc., Odense, Dinamarca).

\section{Análise de dados}

As diversas formas de violência investigadas tiveram as suas frequências apresentadas, porém, para efeito de análise, devido à baixa frequência de alguns tipos, foi criado um indicador único para medir a ocorrência de violência no local de trabalho a partir do agrupamento dessas variáveis. Foi considerada vítima de violência no local de trabalho a pessoa que referiu pelo menos uma dessas modalidades nos últimos 12 meses.

Como variáveis independentes foram consideradas as características individuais e laborais: sexo; idade; cor da pele autorreferida (branca e negra, incluindo preta e parda); escolaridade (até o Nível Médio e Ensino Superior); situação conjugal; categoria profissional [médicos(as), outros profissionais de Nível Superior, auxiliares ou técnicos em enfermagem, e outros profissionais/ trabalhadores até Nível Médio de ensino]; tipo de unidade (hospital de emergência, hospital especializado, nível central - responsável pela formulação das políticas - outros); tipo de atividade (assistencial e não assistencial); e religião no sentido de pertencimento a uma denominação (sim e não). Devido à baixa frequência $(1,63 \%) \mathrm{de}$ amarelos e indígenas, estas categorias não entraram na análise. Em relação à situação conjugal foram utilizadas duas categorias: se unido, sim, caso contrário, não. Após a avaliação das medidas de tendência central, as variáveis contínuas, idade e tempo de serviço, foram convertidas em variáveis categóricas. Quanto à variável tempo de serviço, foram criadas duas categorias: 1 a 19 anos e 20 a 41 anos, e a idade foi recategorizada em três faixas etárias: uma de 25 a 39 anos, e outras duas de 40 a 49 e de 50 a 69 anos.

Para a análise utilizou-se o programa Stata, versão 10.0 (Stata Corp. College Station, Estados Unidos). Realizou-se a análise univariada, descrevendo a distribuição das variáveis dependentes e independentes, geral ou por sexo. Após a apresentação da prevalência global da violência autorreferida no trabalho em saúde, foi investigada a sua associação com as características individuais e laborais. Calculou-se a prevalência do fenômeno em cada um dos estratos, sendo verificadas as associações estimadas pela razão de prevalência (RP) e intervalos de confiança pelo método Mantel Haenszel a 95\%. Por último, procedeu-se à análise multivariada por meio de regressão logística, considerando no modelo as variáveis que apresentaram nível de significância estatística a 5\%. Devido à presença de associação, foram excluídas dessa análise tempo de serviço, que apareceu associado com idade e tipo de atividade, que foram associados com unidade e categoria profissional; também se excluiu escolaridade, associada com cor da pele, sendo que esta última foi priorizada devido à possibilidade da sua influência em episódios envolvendo a discriminação racial. Por outro lado, a variável escolaridade foi contemplada na distribuição das categorias profissionais, ao distinguir profissionais com nível superior daqueles que cursaram até o nível médio de ensino.

\section{Aspectos éticos}

Atendendo aos princípios éticos que regulamentam pesquisas envolvendo seres humanos, o projeto foi previamente analisado e aprovado pelo Comitê de Ética em Pesquisa da SESAB (parecer no 378/2009). Pela assinatura do Termo de Consentimento Livre e Esclarecido buscou-se a anuência dos servidores que eram informados sobre os objetivos, métodos e possíveis benefícios do estudo. Ao mesmo tempo, foram garantidas a confidencialidade e a privacidade das informações.

O trabalho foi divulgado no órgão público por meio de boletim eletrônico interno, além de fôlderes e cartazes que foram distribuídos nas diversas unidades assistenciais e setores da instituição. Cada servidor recebeu uma carta informando sobre a pesquisa e os procedimentos que seriam adotados na produção dos dados.

\section{Resultados}

Foram entrevistados 679 servidores distribuídos entre as 24 unidades assistenciais e a administração central, obtendo-se uma taxa média de resposta de $85 \%$. Das 800 pessoas selecionadas e que atendiam aos critérios de inclusão, 121 recusaram participar do estudo ou não foram localizadas, correspondendo a $4,3 \%$ de recusas e $10,8 \%$ de perdas. Considerando as entrevistas não realizadas, observou-se que entre as mulheres o porcentual foi de $13,5 \%$, e que entre os homens foi de 
21,3\%. Das 86 mulheres não entrevistadas, 31,4\% recusaram, e dentre os homens, 35 não foram entrevistados, com um percentual de recusas de $20 \%$. Em relação às perdas, o percentual entre as mulheres foi de $68,6 \%$ e entre os homens $80 \%$. Assim, entre as perdas e recusas, $71,9 \%$ eram mulheres e a mediana de idade foi de 51 anos (IIQ: 30; 65); em relação ao local de trabalho, $62,8 \%$ estavam lotados em hospitais; quanto à função exercida, 33,0\% eram profissionais da medicina, $34,7 \%$ auxiliares e técnicos em enfermagem e $14 \%$ auxiliares ou técnicos administrativos.

Segundo a Tabela 1, dentre os entrevistados, $81 \%$ eram mulheres e $19 \%$ homens; a maioria residia na capital, porém $9,1 \%$ viviam em cidades do interior do estado, sendo que a mais distante está a $494 \mathrm{~km}$ da capital; a mediana de idade era de 48 anos (IIQ: 40; 54) para ambos os sexos, sendo que $43,3 \%$ das mulheres e $49,6 \%$ dos homens encontravam-se na faixa etária de 50 a 69 anos; em relação à cor da pele, $81 \%$ das mulheres e $88,9 \%$ dos homens consideravam-se negros (pretos e pardos); entre as mulheres o porcentual de graduadas era de $50,5 \%$ e entre os homens de 45,7\%; no que se refere à situação conjugal, 54,4\% das mulheres e $67,4 \%$ dos homens eram unidos.

Em questão de religião, 83,8\% das mulheres e 79,8\% dos homens disseram ter religião ou frequentar algum grupo religioso; em relação ao tempo de serviço, a mediana foi de 19 anos (IIQ: 5 ; 26) para ambos os sexos, de modo que $58,7 \%$ das mulheres e 55,8\% dos homens tinham menos de 1 ano a 19 anos de serviço; no que se refere à função exercida, $32 \%$ das mulheres eram auxiliares ou técnicas em enfermagem, e 31,8\% dos homens trabalhavam como auxiliares ou técnicos administrativos; a maioria das pessoas trabalhava em unidades de emergência, sendo 43,5\% entre as mulheres e $42,6 \%$ entre os homens; o porcentual de mulheres que trabalhavam na assistência era de $62,2 \%$ e entre os homens de $50,4 \%$ (Tabela 1 ).

\section{Padrão de ocorrência de violência autorreferida no local de trabalho}

Apenas $17,8 \%$ das pessoas entrevistadas informaram não ter qualquer preocupação e 23,4\% estavam pouco preocupadas em relação à violência no seu local de trabalho. Quanto às demais, $28,8 \%$ responderam que se sentiam preocupadas, e $30 \%$ muito ou bastante preocupadas; os auxiliares/técnicos em enfermagem foram os mais preocupados $(38,3 \%)$ (dados não apresentados). Ao investigar sobre cada um dos cinco tipos de violência (física, verbal, assédio sexual, discriminação e danos contra a propriedade pessoal) constatou-se que 25,9\% (IC95\%: 22,6\%-
$29,2 \%$ ) dos entrevistados referiram pelo menos uma destas modalidades, sendo a agressão verbal $(19,4 \%)$ a mais frequente, atingindo, especialmente, os auxiliares /técnicos em enfermagem (28\%) e os médicos (23,9\%). Em 66,6\% dos casos a violência aconteceu por mais de duas vezes. Em seguida, aparece a discriminação (5,9\%), destacando-se a racial e a social, baseada na função exercida, entre as mais citadas. Nessa modalidade os auxiliares/técnicos administrativos (9,9\%) foram os mais atingidos. As demais formas apresentaram porcentuais abaixo de $4 \%$ (dados não apresentados).

Homens e mulheres apresentaram uma prevalência global muito próxima para os cinco tipos de violência investigados. Enquanto para os homens a proporção foi de $23,3 \%$, entre as mulheres foi de $26,5 \%$. Ao considerar cada tipo de violência separadamente e por sexo, observou-se não haver diferenças estatisticamente significantes entre homens e mulheres (Figura 1). No que se refere à autoria dos atos de violência, observou-se que em $68 \%$ dos casos os pacientes e seus familiares ocuparam o primeiro lugar. Constatou-se que $28,7 \%$ dos entrevistados também já testemunharam algum tipo de violência no local de trabalho.

Quando analisadas as características sociodemográficas e ocupacionais, por sexo, observou-se que as mulheres entre 25 a 39 anos apresentaram $50,0 \%$ a mais de relato de violência em relação àquelas acima desta faixa etária $(\mathrm{RP}=$ 1,5; IC95\%: 1,1-2,2); as médicas ( $\mathrm{RP}=2,4$; IC95\%: 1,4-4,1) e as auxiliares/técnicas em enfermagem $(\mathrm{RP}=2,1$; IC95\%: 1,3-3,4) referiram duas vezes mais o agravo, quando comparadas a outros profissionais com até Nível Médio de ensino. Entre os homens, observou-se que aqueles entre 25 a 39 anos (RP = 3,1; IC95\%: 1,5-6,1) e com menos de 20 anos de serviço ( $R P=3,2$; IC95\%: 1,4-7,2) referiram três vezes mais violência do que os demais e com maior tempo de serviço; também os homens que trabalhavam em atividades assistenciais referiram mais violência quando comparados aos que desenvolviam outras atividades (RP = 2,3; IC95\%: 1,1-4,8) (Tabela 2).

Após a realização da análise multivariada observou-se que, entre as mulheres, idade e categoria profissional mantiveram associação positiva com violência autorreferida no local de trabalho. As mulheres na faixa etária de 25 a 39 anos apresentaram um acréscimo de $80 \%$ em relação às outras (OR = 1,8; IC95\%: 1,1-3,0), e as médicas também foram as mais atingidas $(\mathrm{OR}=$ 2,5; IC95\%: 1,2-12,5). Entre os homens, ter de 25 a 39 anos (OR = 3,9; IC95\%: 1,9-16,4) e trabalhar como auxiliar ou técnico em enfermagem ( $\mathrm{RP}=$ 3,9; IC95\%: 1,1-13,2) aumentou quase quatro 
Distribuição percentual, por sexo, dos servidores estaduais da saúde, segundo características sociodemográficas e ocupacionais. Salvador, Bahia, Brasil, 2012.

\begin{tabular}{|c|c|c|c|c|}
\hline \multirow[t]{2}{*}{ Características } & \multicolumn{2}{|c|}{ Mulheres $(n=550)$} & \multicolumn{2}{|c|}{ Homens ( $n=129$ ) } \\
\hline & $\mathrm{n}$ & $\%$ & $\mathrm{n}$ & $\%$ \\
\hline \multicolumn{5}{|l|}{ Idade (anos) } \\
\hline $25-39$ & 139 & 25,3 & 27 & 20,9 \\
\hline $40-49$ & 173 & 31,4 & 38 & 29,5 \\
\hline $50-69$ & 238 & 43,3 & 64 & 49,6 \\
\hline \multicolumn{5}{|l|}{ Cor da pele autorreferida } \\
\hline Negra * & 439 & 81,0 & 112 & 88,9 \\
\hline Branca & 103 & 19,0 & 14 & 11,1 \\
\hline \multicolumn{5}{|l|}{ Escolaridade } \\
\hline Até Ensino Médio & 272 & 49,5 & 70 & 54,3 \\
\hline Ensino Superior & 278 & 50,5 & 59 & 45,7 \\
\hline \multicolumn{5}{|l|}{ União conjugal atual } \\
\hline Não & 251 & 45,6 & 42 & 32,6 \\
\hline Sim & 299 & 54,4 & 87 & 67,4 \\
\hline \multicolumn{5}{|l|}{ Religião } \\
\hline Sim & 461 & 83,8 & 103 & 79,8 \\
\hline Não & 89 & 16,2 & 26 & 20,2 \\
\hline \multicolumn{5}{|l|}{ Tempo de serviço no estado (anos) } \\
\hline $1-19$ & 323 & 58,7 & 72 & 55,8 \\
\hline $20-41$ & 227 & 41,3 & 57 & 44,2 \\
\hline \multicolumn{5}{|l|}{ Atividade profissional } \\
\hline Médicos(as) & 61 & 11,1 & 31 & 24,0 \\
\hline Enfermeiros(as) & 58 & 10,5 & 1 & 0,8 \\
\hline Auxiliares/Técnicos em enfermagem & 176 & 32,0 & 21 & 16,2 \\
\hline Auxiliares/Técnicos administrativos & 120 & 21,8 & 41 & 31,8 \\
\hline Outros de Nível Superior & 34 & 6,2 & 22 & 17,1 \\
\hline Outros até Nível Médio & 101 & 18,4 & 13 & 10,1 \\
\hline \multicolumn{5}{|l|}{ Tipo de unidade } \\
\hline Emergência & 239 & 43,4 & 55 & 42,6 \\
\hline Hospitais especializados & 150 & 27,3 & 30 & 23,3 \\
\hline Atenção especial e outros & 83 & 15,1 & 15 & 11,6 \\
\hline Nivel central & 78 & 14,2 & 29 & 22,5 \\
\hline \multicolumn{5}{|l|}{ Tipo de atividade } \\
\hline Assistencial & 342 & 62,2 & 65 & 50,4 \\
\hline Não assistencial & 208 & 37,8 & 64 & 49,6 \\
\hline
\end{tabular}

* Inclui preta e parda.

vezes a ocorrência de violência no trabalho em saúde (Tabela 3).

\section{Discussão}

Este estudo obteve uma taxa de resposta de $85,0 \%$, sendo maior entre as mulheres, resultado considerado satisfatório para uma pesquisa realizada em local de trabalho. A dificuldade em localizar alguns servidores e o receio de que as informações fornecidas pudessem ser utilizadas contra si levaram a $15 \%$ de perdas e recusas. Nesse último caso, a relutância e a desistência em participar foram, algumas vezes, um indício da influência que as pressões político-partidárias ainda exercem sobre o servidor público que teme represálias quando discorda ou tece críticas contra a gestão. 


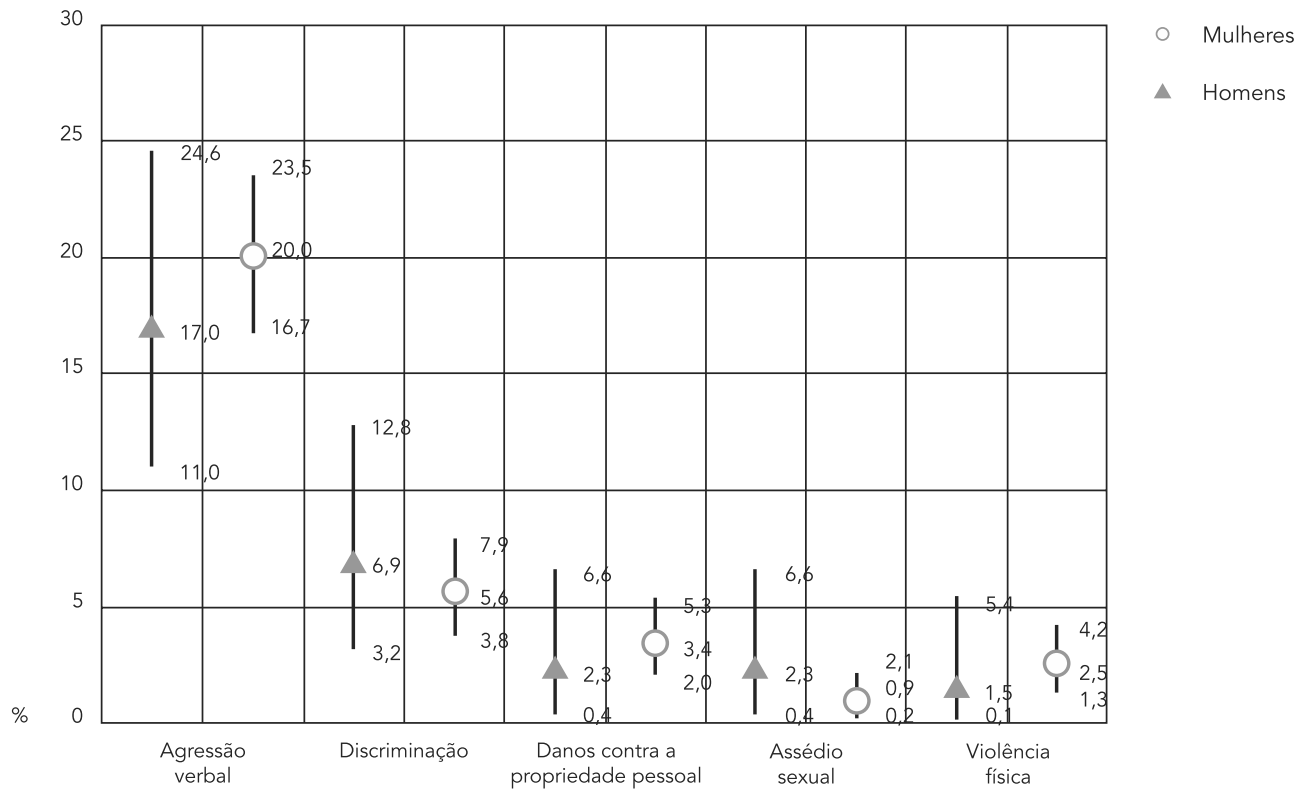

Não se pode afastar algum viés de informação decorrente da omissão de situações de abuso, devendo a prevalência encontrada ser considerada em um patamar mínimo. A agressão verbal foi o tipo mais comum de violência praticada contra os profissionais de saúde, confirmando os achados de outros estudos 11,20,21. As unidades de emergência foram as que apresentaram a maior ocorrência de violência, corroborando a elevada prevalência encontrada em estudos neste tipo de unidade 11,22

O presente estudo não confirmou diferenças estatisticamente significantes entre homens e mulheres ao comparar as diversas modalidades de violência, visto que os intervalos de confiança se superpõem. Entretanto, Palacios et al. 11 consideram que o entendimento do homem sobre o assédio sexual, por exemplo, pode diferir do das mulheres, sendo necessário lembrar que eles também podem sofrer este tipo de abuso, seja do sexo oposto ou de pessoas do mesmo sexo.

Médicos e auxiliares/técnicos em enfermagem foram os que mais referiram violência, sendo, neste caso, confirmadas as diferenças entre os sexos. Entre as mulheres, a maior ocorrência foi entre as médicas, e entre os homens sobressaíram os auxiliares/técnicos em enfermagem.
Essa última categoria é citada em outros estudos como a que mais frequentemente sofre violência no trabalho em saúde e a mais preocupada com o assunto 11,23,24. Em relação à discriminação, os mais atingidos por esta modalidade foram os auxiliares/técnicos administrativos, ou seja, aqueles que não tinham contato direto com o paciente, resultado também encontrado por Palacios et al. 11 .

Durante muitos anos a prática da medicina foi exercida exclusivamente por homens, enquanto que funções julgadas menos importantes, voltadas para a assistência e o cuidado, eram atribuídas às mulheres. Assim, não se pode afastar a possibilidade de que, ainda hoje, concepções que fazem distinção na capacidade de homens e mulheres estejam presentes no imaginário popular, contribuindo para atitudes de desrespeito e discriminação contra profissionais e trabalhadores da saúde.

A violência foi mais prevalente entre as pessoas na faixa etária de 25 a 39 anos, resultado também encontrado por outros autores 12,25,26. Geralmente com menor tempo de serviço, é provável que essas pessoas ainda não detenham a experiência necessária para lidar com as situações de conflito, o que pode acarretar problemas 
Tabela 2

Prevalência e razão de prevalência de violência no trabalho em saúde autorreferida, segundo características sociodemográficas e ocupacionais. Salvador, Bahia, Brasil, 2012.

\begin{tabular}{|c|c|c|c|c|c|c|}
\hline \multirow[t]{2}{*}{ Características } & \multicolumn{3}{|c|}{ Mulheres $(n=550)$} & \multicolumn{3}{|c|}{ Homens $(n=129)$} \\
\hline & $\mathrm{n}$ & $\%$ & RP (IC95\%) & $\mathrm{n}$ & $\%$ & RP (IC95\%) \\
\hline Prevalência global & 550 & 26,5 & - & 129 & 23,3 & - \\
\hline \multicolumn{7}{|l|}{ Idade (anos) } \\
\hline $25-39$ & 139 & 34,5 & $1,5(1,1-2,2)$ & 27 & 48,2 & $3,1(1,5-6,1)$ \\
\hline $40-49$ & 173 & 26,6 & $1,2(0,9-1,7)$ & 38 & 18,4 & $1,2(0,5-2,8)$ \\
\hline $50-69$ & 238 & 21,8 & * & 64 & 15,6 & * \\
\hline \multicolumn{7}{|l|}{ Cor da pele autorreferida } \\
\hline Negra ** & 439 & 28,0 & $1,4(0,9-2,1)$ & 112 & 25,0 & $1,8(0,5-6,6)$ \\
\hline Branca & 103 & 20,3 & * & 14 & 14,2 & * \\
\hline \multicolumn{7}{|l|}{ Escolaridade } \\
\hline Até Ensino Médio & 272 & 25,0 & $0,9(0,7-1,2)$ & 70 & 28,5 & $1,7(0,9-3,3)$ \\
\hline Ensino Superior & 278 & 28,0 & * & 59 & 16,9 & * \\
\hline \multicolumn{7}{|l|}{ União conjugal atual } \\
\hline Não & 251 & 29,0 & $1,2(0,9-1,6)$ & 42 & 28,7 & $1,4(0,7-2,6)$ \\
\hline $\operatorname{Sim}$ & 299 & 24,4 & * & 87 & 20,7 & * \\
\hline \multicolumn{7}{|l|}{ Religião } \\
\hline Sim & 461 & 25,4 & $0,8(0,6-1,1)$ & 103 & 22,3 & $0,8(0,4-1,7)$ \\
\hline Não & 89 & 32,6 & * & 26 & 26,9 & * \\
\hline \multicolumn{7}{|l|}{ Tempo de serviço no estado (anos) } \\
\hline $1-19$ & 323 & 29,4 & $1,3(0,9-1,8)$ & 72 & 33,3 & $3,2(1,4-7,2)$ \\
\hline $20-41$ & 227 & 22,5 & * & 57 & 10,5 & * \\
\hline \multicolumn{7}{|l|}{ Atividade profissional } \\
\hline Médicos(as) & 61 & 37,7 & $2,4(1,4-4,1)$ & 31 & 12,9 & $0,4(0,1-1,4)$ \\
\hline Enfermeiros(as) & 58 & 25,8 & $1,6(0,9-3,0)$ & $1 \star \star \star$ & - & - \\
\hline Auxiliares/Técnicos em enfermagem & 175 & 33,1 & $2,1(1,3-3,4)$ & 21 & 52,4 & $1,7(0,7-4,2)$ \\
\hline Auxiliares/Técnicos administrativos & 120 & 22,5 & $1,3(0,8-2,2)$ & 41 & 17,0 & $0,7(0,3-1,9)$ \\
\hline Outros de Nível Superior & 34 & 20,5 & $1,3(0,6-2,9)$ & 22 & 18,1 & $0,6(0,2-1,9)$ \\
\hline Outros até Nível Médio & 102 & 15,7 & * & 13 & 30,7 & * \\
\hline \multicolumn{7}{|l|}{ Tipo de unidade } \\
\hline Emergência & 239 & 30,5 & $1,7(1,0-2,8)$ & 55 & 34,5 & $2,5(0,9-6,7)$ \\
\hline Hospitais especializados & 150 & 28,0 & $1,6(0,9-2,7)$ & 30 & 13,8 & $1,2(0,4-4,1)$ \\
\hline Atenção especial e outros & 83 & 20,4 & $1,1(0,6-2,2)$ & 15 & 13,3 & $1,0(0,2-4,7)$ \\
\hline Nível central & 78 & 17,9 & * & 29 & 13,7 & $*_{-}$ \\
\hline \multicolumn{7}{|l|}{ Tipo de atividade } \\
\hline Assistencial & 342 & 30,1 & $1,5(1,0-2,0)$ & 70 & 32,3 & $2,3(1,1-4,8)$ \\
\hline Não assistencial & 208 & 20,7 & * & 59 & 14,1 & * \\
\hline
\end{tabular}

IC95\%: intervalo de 95\% de confiança; RP: razão de prevalência.

* Valor de referência;

** Excluídos desta análise 1,63\% de indígenas, amarelos e uma recusa à questão; negra inclui preta e parda;

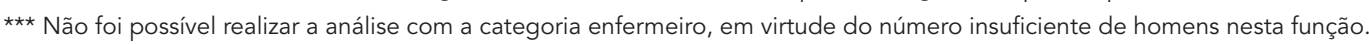

nas relações interpessoais. Por outro lado, as pessoas mais jovens, geralmente, estão em posição de desvantagem na hierarquia social, o que as tornam mais vulneráveis a sofrer abusos de superiores ou colegas mais experientes.
De modo que, na análise estratificada, observou-se que a variável tempo de serviço estava associada com a ocorrência do fenômeno, sendo a violência, autorreferida, três vezes mais prevalente em homens com menos de 20 anos 
Fatores associados à violência no trabalho autorreferida entre servidores estaduais da saúde, conforme análise de regressão logística, por sexo. Salvador, Bahia, Brasil, 2012.

\begin{tabular}{|c|c|c|}
\hline \multirow[t]{2}{*}{ Características } & Mulheres $(n=550)$ & Homens $(n=129)$ \\
\hline & OR (IC95\%) & OR (IC95\%) \\
\hline \multicolumn{3}{|l|}{ Idade (anos) } \\
\hline $25-39$ & $1,8(1,1-3,0)$ & $3,9(1,2-12,5)$ \\
\hline $40-49$ & $1,3(0,8-2,1)$ & $0,8(0,2-2,7)$ \\
\hline $50-69$ & * & * \\
\hline \multicolumn{3}{|l|}{ Cor da pele autorreferida } \\
\hline Negra ** & $1,4(0,8-2,6)$ & $1,6(0,2-9,8)$ \\
\hline Branca & * & * \\
\hline \multicolumn{3}{|l|}{ União conjugal atual } \\
\hline Não & $1,3(0,9-2,0)$ & $1,2(0,4-3,4)$ \\
\hline $\operatorname{Sim}$ & * & * \\
\hline \multicolumn{3}{|l|}{ Crença religiosa } \\
\hline Não & $1,2(0,7-2,1)$ & $1,0(0,3-3,2)$ \\
\hline Sim & * & * \\
\hline \multicolumn{3}{|l|}{ Atividade profissional } \\
\hline Médicos(as) & $2,5(1,2-5,2)$ & $0,5(0,1-6,1)$ \\
\hline Auxiliares/Técnicos em enfermagem & $1,5(0,8-2,6)$ & $3,9(1,1-13,2)$ \\
\hline Outros profissionais de Nível Superior & $0,8(0,4-1,5)$ & $1,2(0,2-6,1)$ \\
\hline Outros profissionais até Nível Médio & * & * \\
\hline \multicolumn{3}{|l|}{ Tipo de atividade } \\
\hline Assistencial & $0,6(0,3-1,2)$ & $0,7(0,1-2,5)$ \\
\hline Não assistencial & * & \\
\hline
\end{tabular}

IC95\%: intervalo de 95\% de confiança; OR: odds ratio.

* Categoria de referência;

** Inclui preta e parda.

de serviço. Entretanto, esse resultado difere do estudo com médicos de unidades de prontoatendimento, onde os mais atingidos foram os profissionais com maior tempo de admissão 22 .

O baixo poder amostral entre os homens pode ter concorrido para que eventos com pouca ocorrência não fossem detectados. Não obstante, acredita-se que o estudo possa trazer contribuições importantes para a visibilidade da violência no setor saúde e fornecer subsídios para a formulação de políticas de atenção à saúde dos trabalhadores. Entende-se que todas as medidas adotadas no sentido de melhorar as condições de trabalho dos profissionais da saúde terão repercussão na qualidade do atendimento prestado à população.

Aspectos que são determinantes na ocorrência dos agravos, como as características organizacionais e o processo de trabalho em saúde, devem ser incluídos nas investigações, contribuindo, assim, para um maior entendimento da violência no trabalho em saúde e para a busca de estratégias para combatê-la.

Investigações que contemplem as populações assistidas poderão trazer elementos para o entendimento das expectativas em relação ao serviço que lhes é prestado, identificando falhas no sistema de saúde que comprometem a relação entre profissional e usuário. Cabe, ainda, uma reflexão sobre a formação do profissional e trabalhador da saúde, na busca de maior articulação entre trabalho, ensino e serviço, de modo a garantir a capacitação de pessoas comprometidas com uma assistência de melhor qualidade e em condições de lidar com situações de conflito decorrentes das diferenças sociais, econômicas e culturais. 


\section{Resumen}

Este estudio tuvo como objetivo estimar la prevalencia de la violencia entre los trabajadores de la salud. Es un estudio transversal con una muestra de 679 trabajadores del estado de Bahía, Brasil, donde se usó un cuestionario mediante entrevistas. De los encuestados, el 25,9\% (IC95\%: 22,6\%-29,2\%) informó al menos de una de las formas de violencia investigada, donde la agresión verbal (19,4\%) era la más frecuente. A las mujeres de 25 a 39 años les afectó un aumento del 80\% en la incidencia de la violencia (OR = 1,8; IC95\%: 1,1-3,0), mientras que los médicos también fueron los más afectados (OR = 2,5; IC95\%: 1.2-12.5). Entre los hombres, que tienen entre 25 y 39 años (OR = 3,9; IC95\%: 1,916,4) y trabajan como asistentes de enfermería o técnicos (RP = 3,9; IC95\%: 1,1-13,2) aumentó casi 4 veces la incidencia de violencia en el trabajo en salud. Este estudio puede contribuir de manera significativa a la visibilidad de la violencia en el sector de la salud y servir de apoyo para la formulación de la política de atención a los trabajadores que repercute en la calidad de la atención prestada.

Violencia Laboral; Personal de Salud;

Identidad de Género

\section{Colaboradores}

I. V. Silva contribuiu com a revisão da literatura, definição do desenho de estudo, elaboração do instrumento de coleta de dados, supervisão e participação na produção e sistematização dos dados, análise e interpretação de resultados e redação do artigo. E. M. L. Aquino participou da concepção do estudo, análise e interpretação de resultados e redação do artigo. I. C. M. Pinto foi responsável pelo projeto de pesquisa junto à instituição financiadora, participou da concepção do estudo, coordenação da produção dos dados e revisão da redação do artigo.

\section{Agradecimentos}

À FAPESB que financiou este estudo.

\section{Referências}

1. Contrera-Moreno L, Contrera-Moreno MI. Violência no trabalho em enfermagem: um novo risco ocupacional. Rev Bras Enferm 2004; 57:746-9.

2. World Health Organization. Global consultation on violence and health. Violence: a public health priority. Geneva: World Health Organization; 1996 (Document WHO/EHA/SPI.POA.2).

3. Leather P. Workplace violence: scope, definition and global context. In: Cooper CL, Swanson N, editors. Workplace violence in the health sector: state of the art. Geneva: International Labour Office; 2002. p. 3-18.

4. Santos Junior EA, Dias EC. Violência no trabalho: uma revisão de literatura. Rev Bras Med Trab 2004; 2:36-54.

5. Cooper CL, Swanson N, editors. Workplace violence in the health sector: state of the art. Geneva: World Health Organization; 2002.

6. Ferrinho P, Biscaia A, Fronteira I, Craveiro I, Antunes AR, Conceição C, et al. Patterns of perceptions of workplace violence in the Portuguese health care sector. Hum Resour Health 2003; I:11.

7. Curbow B. Origins of violence at work. In: Cooper CL, Swanson N, editors. Workplace violence in the health sector: state of the art. Geneva: World Health Organization; 2002. p. 35-48.
8. Deslandes SF, Gomes R, Silva CMFP. Caracterização dos casos de violência doméstica contra a mulher atendidos em dois hospitais públicos do Rio de Janeiro. Cad Saúde Pública 2000; 16:129-37.

9. Schraiber LB, d'Oliveira AFPL, França-Junior I, Pinho A. Violência contra a mulher: estudo em uma unidade de atenção primária à saúde. Rev Saúde Pública 2002; 36:470-7.

10. d'Oliveira AFPL, Schraiber LB, França-Junior I, Ludermir AB, Portella AP, Diniz CS, et al. Fatores associados à violência por parceiro íntimo em mulheres brasileiras. Rev Saúde Pública 2009; 43:299311.

11. Palacios M, Santos ML, Val MB, Medina M, Abreu M, Cardoso LS, et al. Workplace violence in the health sector: country case study - Brazil. Geneva: ILO/ICN/WHO/PSI Joint Programme on Workplace Violence in the Health Sector; 2002.

12. Franz S, Zeh A, Schablon A, Kuhnert S, Nienhaus A. Aggression and violence against health care workers in Germany - a cross sectional retrospective survey. BMC Health Serv Res 2005; 10:51. 
13. Gerberich SG, Church T, McGoven P, Hansen H Nachreiner N, Geisser M, et al. An epidemiological study of the magnitude and consequences of work related violence: the Minnesota Nurses' Study. Occup Environ Med 2004; 61:495-503.

14. Jackson M, Ashley D. Physical and psychological violence in Jamaica's health sector. Rev Panam Salud Pública 2005; 18:114-21.

15. Ferrinho P, coordenador. Violência no local de trabalho no sector da saúde: estudos de caso portugueses. Lisboa: Associação para o Desenvolvimento e Cooperação Garcia de Orta; 2002.

16. Mendes-Gonçalves RB. Práticas de saúde: processos de trabalho e necessidades. São Paulo: CEFOR; 1992. (Cadernos CEFOR - Textos 1).

17. Deslandes SF. Frágeis deuses: profissionais da emergência entre os danos da violência e a recriação da vida. Rio de Janeiro: Editora Fiocruz; 2002.

18. Batista CB, Campos AS, Reis JC, Schall VT. Violência no trabalho em saúde: análise em unidades básicas de saúde de Belo Horizonte, Minas Gerais. Trab Educ Saúde 2011; 9:295-317.

19. Lancman S, Sznelwar LI, Uchida S, Tuacek TA. O trabalho na rua e a exposição à violência no trabalho: um estudo com agentes de trânsito. Interface Comun Saúde Educ 2007; 11:79-92.

20. Menckel E, Viitasara E. Threats and violence in Swedish care and welfare - magnitude of the problem and impact on municipal personnel. Scand J Caring Sci 2002; 16:376-85.
21. Cezar ES, Marziale MHP. Problemas de violência ocupacional em um serviço de urgência hospitalar da Cidade de Londrina. Cad Saúde Pública 2006; 22:217-21.

22. Santos Junior EA, Dias EC. Médicos vítimas da violência no trabalho em unidades de pronto atendimento. Cad Saúde Colet (Rio J) 2005; 13:705-22.

23. Campos AS, Dias EC. Perfil de agressões em unidades básicas de Belo Horizonte - MG: é possível pensar no processo de trabalho. Rev Méd Minas Gerais 2006; 16(4 Suppl 2):80-5.

24. Xavier ACH, Barcelos CRV, Lopes JP, Chamarelli PG, Ribeiro SS, Lacerda LS, et al. Assédio moral no trabalho no setor saúde no Rio de Janeiro: algumas características. Rev Bras Saúde Ocup 2008; 33:15-22.

25. Wu S, Zhu W, Li H, Lin S, Chai W, Wang X. Workplace violence and influencing factors among medical professionals in China. Am J Ind Med 2012; 55:1000-8.

26. Magnavita N. The exploding spark: workplace violence in an infectious disease hospital - a longitudinal study. Biomed Res Int 2013; 2013:316358.

Recebido em 12/Ago/2013

Versão final reapresentada em 07/Mar/2014

Aprovado em 18/Mar/2014 